07

\title{
Управление свойствами спин-волнового транспорта в полукольцевом магнонном микроволноводе
}

\author{
(C) В.А. Губанов, А.А. Мартышкин, С.Е. Шешукова, А.В. Садовников \\ Саратовский государственный университет им. Н.Г. Чернышевского, \\ 410012 Саратов, Россия \\ e-mail: vladmeen@gmail.com
}

Поступило в Редакцию 28 марта 2019 г.

В окончательной редакции 28 марта 2019 г.

Принято к публикации 15 апреля 2019 г.

Исследован спин-волновой транспорт вдоль волноведущей структуры с нарушением трансляционной симметрии. Структура на основе пленки железо-иттриевого граната образована полукруглым участком магнитного микроволновода. Показано, что при вариации угла подмагничивания в плоскости микроволновода оказывается возможным управлять пространственным распределением динамической намагниченности. При этом наблюдается значительное изменение коэффициента прохождения спиновой волны. Предложенная структура позволяет осуществить поворот спин-волновых сигналов в нерегулярной структуре в режиме распространения поверхностной магнитостатической волны, что может быть использовано в планарных топологиях магнонных сетей.

Ключевые слова: спиновые волны, магноника, межсоединения, магнитостатические волны, нерегулярный волновод.

DOI: $10.21883 /$ JTF.2019.11.48335.147-19

\section{Введение}

В настоящее время большой интерес представляет исследование физических принципов, определяющих возможность использования спиновых волн $(\mathrm{CB})$ для создания устройств обработки информационного сигнала на принципах магноники [1-4], при этом в одним из наиболее перспективных материалов для создания функциональных структур в СВЧ-диапазоне длин волн являются тонкие пленки железо-иттриевого граната (ЖИГ) с рекордно низкой величиной затухания $\mathrm{CB}$. В касательно намагниченных однородных тонких ферромагнитных пленках возможны распространения магнитостатических волн двух типов. В случае направления распространения волны поперечно направлению поля подмагничивания в пленке распространяются поверхностные магнитостатические спиновые волны (ПМСВ) [5]. Если же равновесное направление намагниченности коллинеарно направлению распространения волны, то в пленке распространяются обратные объемные магнитостатические волны (ООМСВ), характеризуемые противоположным направлением групповой и фазовых скоростей [6-8]. Для случая тонкой пленки с соизмеримыми геометрическими размерами в латеральной плоскости частотные спектры ПМСВ и ООМСВ не перекрываются, т. е. в случае заданной частоты СВ при соответствующей ориентации внешнего магнитного поля в пленке может распространяться только один тип волн $[6,7,9,10]$. Однако в структурах с нарушенной трансляционной симметрией возможным оказывается ситуация, при которой один тип волны может преобразовываться в другой ввиду наличия сильно выраженной анизотропии формы магнитной микроструктуры $[11,12,13]$. При этом направление распространения спиновой волны меняется на ортогональное, т. е. волна поворачивает на $90^{\circ}$. Однако для планарных топологий магнонных сетей $[14,15]$ важным оказывается рассмотрение случая изменения направления распространения СВ на противоположное. Добиться этого оказывается возможным, например, используя одномерную магнонно-кристаллическую структуру $[16,19]$, представляющую собой среду с периодическим изменением параметров (намагниченность, толщина пленки, ширина микроволновода), в которой при выполнении условия резонанса Брэгга [17] может формироваться $\mathrm{CB}$, распространяющаяся в направлении, противоположном направлению распространения волны, падающей на периодическую структуру. Однако в этом случае оказывается возможным только распространение волны с противоположно направленной групповой скоростью вместе с падающей на магнонный кристалл (МК) волной, что затрудняет использование такой структуры в качестве функционального блока магнонной сети [18]. Другой альтернативой решения проблемы „поворота“ СВ может быть система латерально связанных МК [19], в которой удается организовать направленное ответвление спин-волновой мощности как в направлении распространения $\mathrm{CB}$, возбуждаемой в одном МК, так и в противоположном направлении $[19,20]$. Ввиду особенностей частотно-селективных режимов спин-волновой связи в таких структурах изменение направления распространения СВ на противоположное возможно только в узкой полосе частот входного сигнала. Одним из возможных путей преодоления обозначенных ограничений является создание структур с нарушением трансляционной сим- 
метрии вдоль направления распространения $\mathrm{CB}$, при этом форма структуры может представлять собой сегмент кольца, ширина которого совпадает с шириной магнонного микроволновода, в котором возбуждается CB [21-23], однако механизм распространения СВ в таких структурах не был детально исследован.

В настоящей работе с помощью метода микромагнитного моделирования показана возможность использования структуры с нарушением трансляционной симметрии для разворота направления распространения спин-волнового сигнала на противоположное. Детально исследовано распределение динамической намагниченности в области полукольца, изготовленного из тонкой пленки ЖИГ. Проведен расчет характеристик спин-волнового транспорта при изменении направления и величины поля подмагничивания.

\section{Исследуемые структуры и методика численного моделирования}

Исследуемая структура на основе тонкопленочного нерегулярного магнонного микроволновода представляла собой сегмент кольца шириной $w=500 \mu \mathrm{m} \mathrm{c}$ внутренним радиусом $r=500 \mu \mathrm{m}$. На рис. $1, a$ показана схема структуры типа „полукольцо“, образованной из пленки ЖИГ [(YIG) $\left.\mathrm{Y}_{3} \mathrm{Fe}_{5} \mathrm{O}_{12}(111)\right]$ толщиной $d_{1}=10 \mu \mathrm{m}$ с намагниченностью насыщения $M_{0}=1.39 \cdot 10^{5} \mathrm{~A} / \mathrm{m}$. Пленка ЖИГ расположена на подложке из галлийгадолиниевого граната [(GGG) $\mathrm{Gd}_{3} \mathrm{Ga}_{5} \mathrm{O}_{12}$ (111)] толщиной $d_{2}=500 \mu \mathrm{m}$. Константа неоднородного обмена пленки ЖИГ полагалась равной $A_{e x}=3.614 \cdot 10^{-12} \mathrm{~J} / \mathrm{m}$. Направление внешнего однородного магнитного поля, равного $\left|\mathbf{H}_{0}\right|=1200$ Ое, совпадало с положительным направлением оси $x$, что обеспечивало эффективное возбуждение ПМСВ.

Аналитическое описание распространения СВ вдоль волновода с нарушением трансляционной симметрии, как правило, невозможно сформулировать без существенных приближений $[24,25]$. Поэтому для описания динамики СВ в рассматриваемой структуре был использован метод численного микромагнитного моделирования на основе решения Ландау-Лифшица-Гильберта [26-28]:

$$
\frac{\partial \mathbf{M}}{\partial t}=\mathcal{\gamma}\left[\mathbf{H}_{\mathrm{eff}} \times \mathbf{M}\right]+\frac{\alpha}{M_{0}}\left[\mathbf{M} \times \frac{\partial \mathbf{M}}{\partial t}\right],
$$

где $\mathbf{M}$ - вектор намагниченности, $\alpha=10^{-5}-$ параметр затухания, $\mathbf{H}_{\mathrm{eff}}=-\frac{\delta F}{\delta \mathbf{M}}-$ эффективное магнитное поле, $F$ - свободная энергия магнетика. Вкладом магнитокристаллической анизотропии в плоскости пленки ввиду его малости можно пренебречь. На рис. 1, а показана только область полукольца, однако в численном эксперименте в плоскости $S_{1}$ структуры было рассмотрено распределение намагниченности, созданное распространяющейся вдоль отрицательного направления оси у ПМСВ вдоль

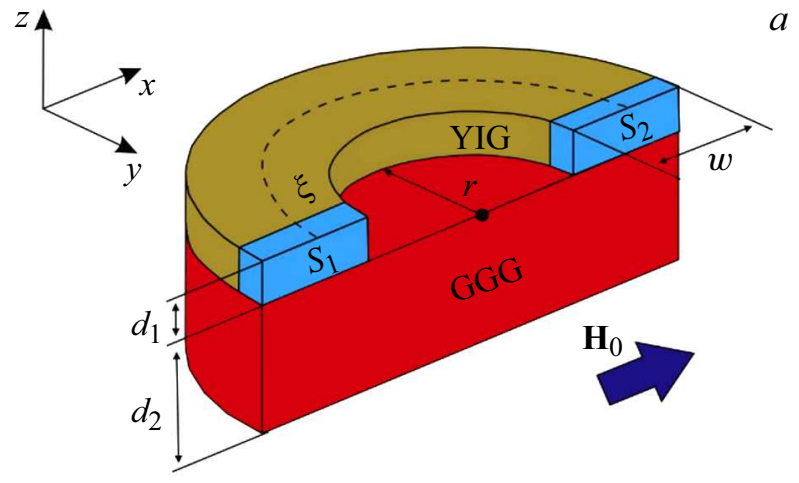

$a$
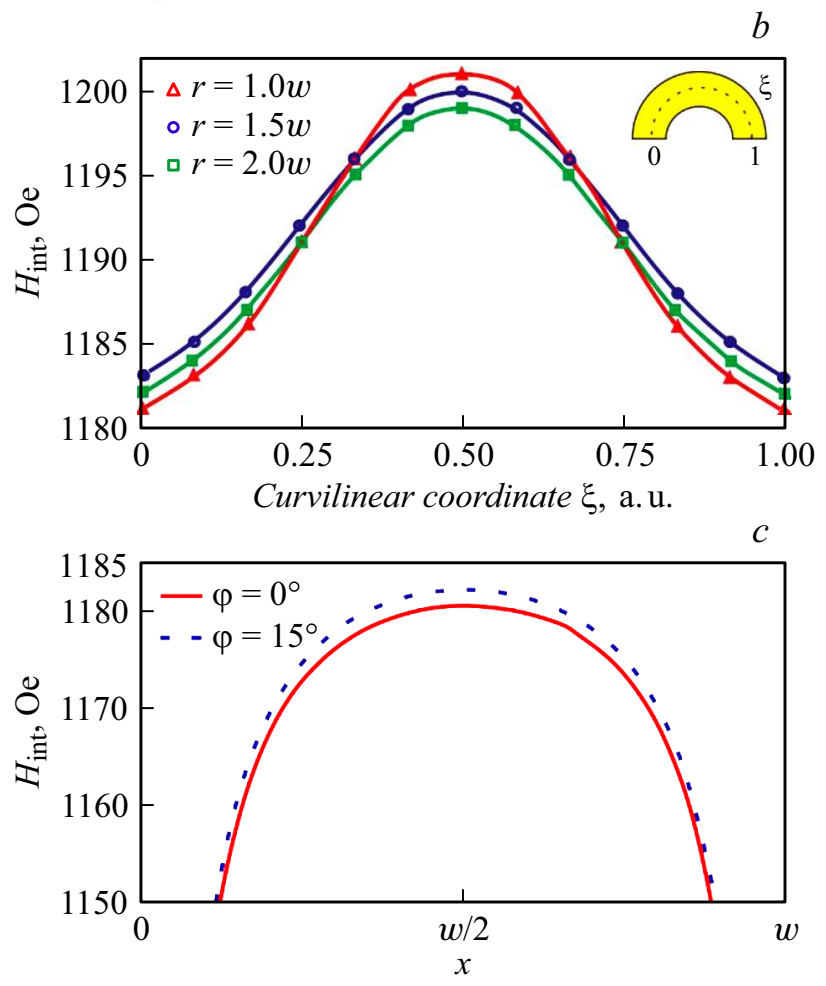

Рис. 1. $a-$ схема исследуемой структуры; $b-$ величина внутреннего магнитного поля $H_{\text {int }}$ как функция криволинейной координаты $\xi$ для случаев $r=1 w$ (треугольники), $r=1.5 w$ (кружки), $r=2 w$ (квадраты). На вставке показана криволинейная система координат при $0<\xi<1 ; c-$ распределение $H_{\text {int }}(x)$ для области подводящего микроволновода в случае угла подмагничивания $\varphi=0^{\circ}$ (сплошная кривая) и $\varphi=-15^{\circ}$ (пунктирная кривая).

подводящего ЖИГ микроволновода шириной $w$ и толщиной $d_{1}$. В плоскости $S_{2}$ полагалось, что полукольцо нагружено на микроволновод шириной $w$ и толщиной $d_{1}$, что исключало влияние отраженной СВ на динамику СВ в области полукольца.

В магнонных микроволноводах ввиду анизотропии формы распределение внутреннего поля может быть сильно неоднородным, что может приводить, например, к формированию краевых магнонных мод [29, 30]. На рис. $1, b$ представлена зависимость внутреннего магнитного поля $H_{\text {int }}$ вдоль криволинейной координаты $\xi$ 

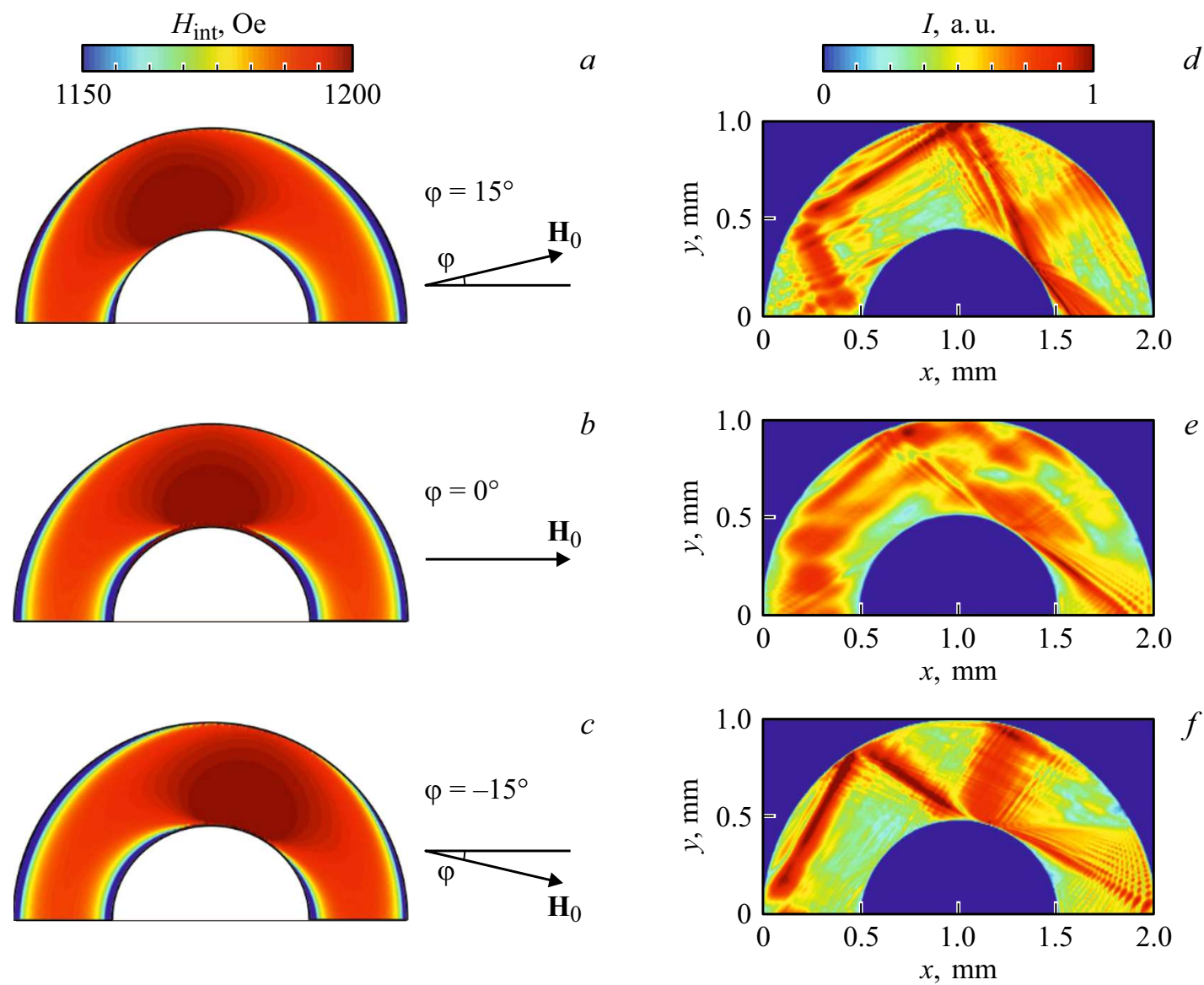

Рис. 2. Профили внутреннего магнитного поля $(a-c)$ и интенсивность распространяющейся СВ на частоте $f=5.20 \mathrm{GHz}(d-f)$ в полукольцевом микроволноводе для различных значений углов подмагничивания: $\varphi=15^{\circ}, 0^{\circ},-15^{\circ}$ (отмечены на рисунке).

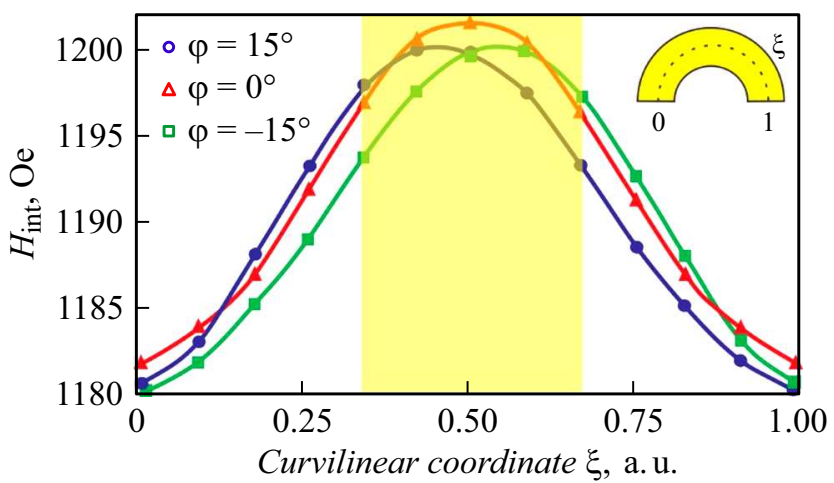

Рис. 3. Величина внутреннего магнитного поля как функция криволинейной координаты $\xi$ для угла подмагничивания $\varphi=15^{\circ}$ (кружки), $0^{\circ}$ (треугольники), $-15^{\circ}$ (квадраты). На вставке показана криволинейная система координат при $0<\xi<1$.

(параметр нормирован на единицу) для полуколец с радиусами $r=1 w, 1.5 w$ и $2 w$. Видно, что профиль внутреннего магнитного поля $H_{\text {int }}$ имеет явно выраженный максимум в центре полукольца при значении $\xi=0.5$, что обусловлено анизотропией формы. При этом зна- чения величины $H_{\text {int }}$ различны на краях полукольца в плоскости $S_{1}$ и $S_{2}$ при $\xi=0$ и 1 соответственно. На рис. 1, $c$ показано распределение внутреннего поля вдоль оси $x$ в подводящем ЖИГ микроволноводе шириной $w$ и толщиной $d_{1}$. Видно, что значение внутреннего поля $H_{\text {int }}(x=w / 2) \cong H_{\text {int }}(\xi=0) \cong H_{\text {int }}(\xi=1)$ в случае $r=1 w$. Также для указанного значения внутреннего радиуса полукольца величина $\delta_{H}=$ $=H_{\text {int }}(\xi=0.5)-H_{\text {int }}(\xi=0)$ имеет наибольшее значение, что обусловливает наибольшую величину диапазона перекрытия дисперсионных характеристик ПМСВ и ООМСВ [31] и, следовательно, расширяет частотный диапазон, в котором достигается эффективная передача мощности СВ в плоскость $S_{2}$.

Для определения характеристик спин-волнового транспорта в рассматриваемой структуре следом за расчетом распределения статической намагниченности решалась задача о возбуждении и распространении ПМСВ в области подводящего волновода и далее трансформации спин-волнового сигнала в области нерегулярной структуры - микроволновода в виде полукольца. Стоит отметить, что в данной структуре в отличие от связанных волноводов [32] „провисающие“ динамические магнитные поля подводящих микроволноводов не вза- 

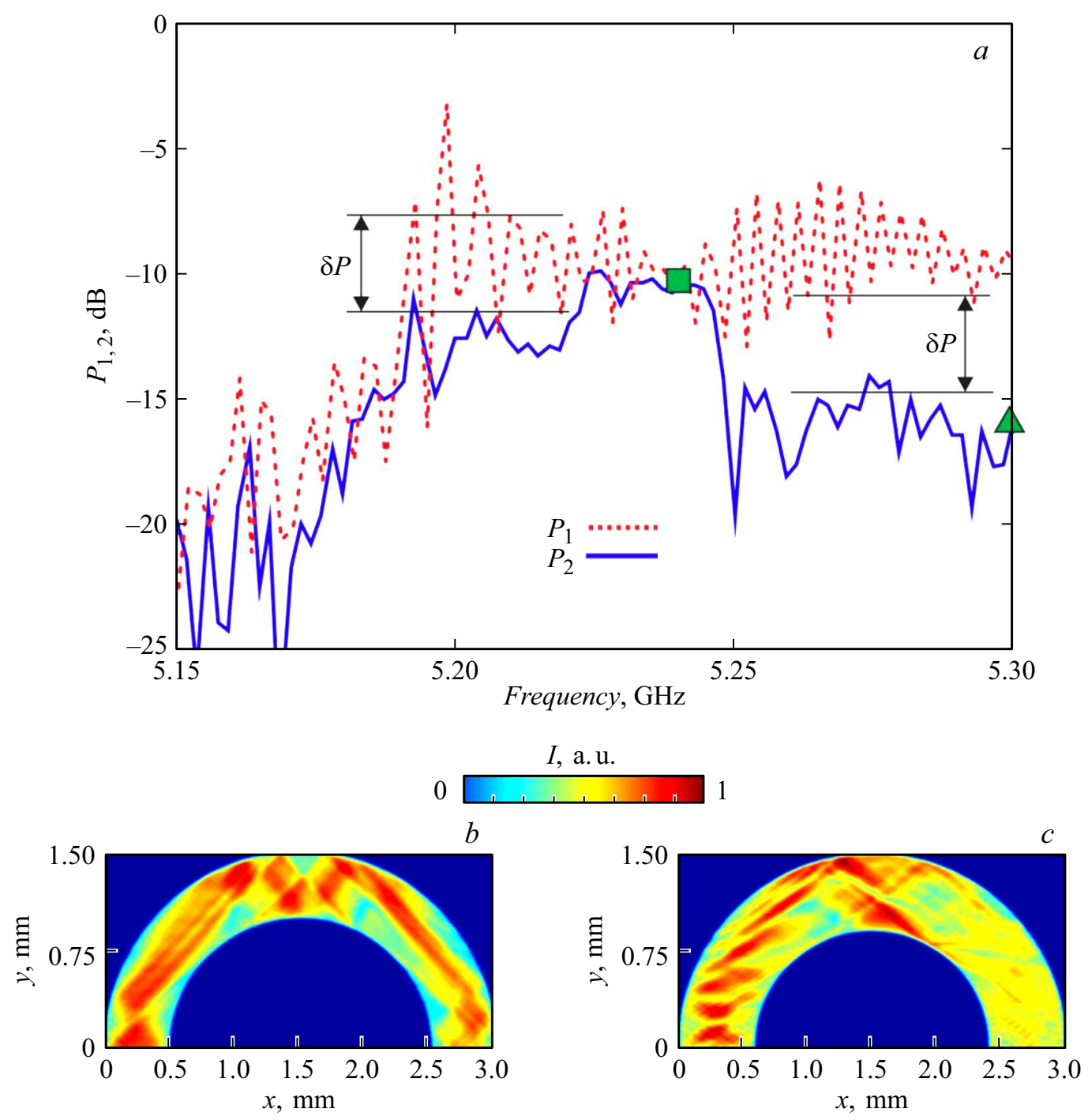

Рис. 4. Мощность спин-волнового сигнала в сечениях $S_{1}$ и $S_{2}$ в полукольцевом магнонном микроволноводе радиусом $r=2 w(a)$; пространственное распределение интенсивности СВ на частоте $f=5.24 \mathrm{GHz}$ (квадрат на рис. $4, a)(b)$ и $f=5.3 \mathrm{GHz}($ треугольник на рис. $4, a)(c)$.

имодействуют друг с другом ввиду выполнения условия $2 r \gg d_{1}$.

Рассмотрим трансформацию распределения внутреннего магнитного поля и интенсивности СВ $I=$ $=\sqrt{m_{y}^{2}+m_{z}^{2}}$ на частоте $f=5.2 \mathrm{GHz}$ для случаев разной ориентации внешнего подмагничивающего поля $\mathbf{H}_{0}$ относительно положительного направления оси $x$ (рис. 2). При отклонении $\mathbf{H}_{0}$ на угол $\varphi=15^{\circ}$ наблюдалось увеличение величины $I$ в выходной плоскости $S_{2}$ по сравнению со случаем $\varphi=0^{\circ}$. Однако при $\varphi=-15^{\circ}$ значение интенсивности СВ на выходе $S_{2}$ уменьшалось (ср. рис. 2, $d-f$ ).

Для объяснения наблюдаемого изменения интенсивности СВ были построены зависимости внутреннего поля на оси нерегулярной структуры для рассматриваемых значений угла подмагничивания (рис. 3).
Видно, что при отклонении поля подмагничивания от положительного направления оси $x$ положение максимума величины $H_{\text {int }}$ смещается от точки $\xi=0.5$ вправо при $\varphi=-15^{\circ}$, либо влево при $\varphi=15^{\circ}$. Стоит отметить, что при изменении угла подмагничивания также изменяется значение внутреннего поля на оси подводящего волновода (рис. 1, 1 ).

Таким образом, трансформация ПМСВ, распространяющейся в нерегулярной области полукольцевого микроволновода, в ООМСВ происходит при меньших значениях $\xi$ в случае $\varphi=15^{\circ}$, чем при $\varphi=0^{\circ}$ либо $\varphi=-15^{\circ}$. При этом область, в которой ожидается распространение ООМСВ при $\varphi=0^{\circ}$ показана на рис. 3 заливкой при $0.33<\xi<0.66$. С другой стороны, на рис. $2, d$ видно, что луч СВ при $\xi>0.5$ скользит вдоль нерегулярной секции полукольца, испытывая меньшее отражение от границы, чем в случае $\varphi=0^{\circ}$ (рис. 2,e), 
$\varphi=-15^{\circ}$ (рис. $\left.2, f\right)$, что может объяснить изменение интенсивности СВ в плоскости $S_{2}$.

В рассматриваемой структуре оказывается возможным реализация режимов пространственно-частотной селекции СВ. На рис. 4, $a$ показан результат расчета спектра мощности выходного спин-волнового сигнала $P_{1,2}(f)$ в плоскости $S_{1,2}$. Частотная зависимость мощности сигнала в плоскости $S_{1}$ имеет изрезанный профиль ввиду, во-первых, близости плоскости $S_{1}$ к источнику, а, во-вторых, наличия сильных отражений сигнала в области сочленения подводящего волновода и нерегулярной секции. Видно, что в полосе пропускания рассматриваемой структуры $5.18 \mathrm{GHz}<f<5.35 \mathrm{GHz}$ на спектре прохождения СВ можно выделить выраженные максимумы и минимумы. Причем можно отметить изменение в величине мощности на $\delta P \sim 4 \mathrm{~dB}$ при прохождении спин-волновым сигналом полукруглой области нерегулярной структуры. При частоте входного сигнала $f=5.24 \mathrm{GHz}$ наблюдается эффективная передача мощности СВ от плоскости $S_{1}$ к плоскости $S_{2}$ (рис. 4,b). При этом можно отметить симметричный профиль распределения интенсивности СВ относительно $\xi=0.5$. На частоте $f=5.3 \mathrm{GHz}$ в области $\xi=0.5$ наблюдается затухание СВ по мере распространения к плоскости $S_{2}$ (рис. $4, c$ ). Отметим, что величина разности внутренних полей в центре подводящего микроволновода $H_{\mathrm{int}}(x=w / 2)$ и в полукольце при $\xi=0.5$ составляла $H_{\text {int }}(\xi=0.5)-H_{\text {int }}(x=w / 2)=20$ Ое, что позволяет ожидать частотный диапазон перекрытия ПМСВ и OOMCB $\Delta f=\gamma \Delta H_{\text {int }}=56 \mathrm{MHz}(\gamma=2.8 \mathrm{MHz} / \mathrm{Oe}$ - гиромагнитное отношение для ЖИГ), однако при учете конечной ширины ЖИГ микроволноводов [33] частотный диапазон перекрытия соответствующих дисперсионных характеристик увеличивается [31].

\section{Заключение}

Таким образом, используя методы микромагнитного моделирования, было показано что структура с нарушением трансляционной симметрии в виде полукольца может использоваться в планарных топологиях магнонных сетей для разворота направления распространения спин-волнового сигнала на противоположное. При этом вариация угла подмагничивания приводит к изменению эффективности передачи спин-волнового сигнала и изменению коэффициента прохождения спиновой волны, равного отношению мощности сигнала на различных концах полукруглого микроволновода. Последнее обусловлено влиянием анизотропии формы нерегулярной структуры на распространение спиновых волн в области полукольца. Предложенная структура позволяет осуществлять пространственно-частотную селекцию спин-волновых сигналов, что позволяет использовать ее в качестве функционального элемента межсоединений в системах обработки информационного сигнала.

\section{Финансирование работы}

Расчет волновых характеристик и распределения динамической намагниченности и модового состава спиновых волн, распространяющихся в $U$-образном магнитном элементе, выполнен в рамках проекта Российского Научного Фонда (№ 18-79-00198). Исследование угловых зависимостей распространения спиновых волн выполнено в рамках гранта Российского фонда фундаментальных исследований (№ 18-37-20005) и гранта президента Российской Федерации (МК-3650.2018.9).

\section{Конфликт интересов}

Авторы заявляют, что у них нет конфликта интересов.

\section{Список литературы}

[1] Kruglyak V.V., Demokritov S.O., Grundler D. // J. Phys. D: Appl. Phys. 2010. Vol. 43. P. 264001.

[2] Demidov V.E., Urazhdin S., Zholud A., Sadovnikov A.V., Slavin A.N., Demokritov S.O. // Sci. Rep. 2015. Vol. 5. P. 8578.

[3] Demidov V.E., Urazhdin S., De Loubens G., Klein O., Cros V., Anane A., Demokritov S.O. // Phys. Rep. 2017. Vol. 673. P. $1-31$.

[4] Sadovnikov A.V., Grachev A.A., Sheshukova S.E., Sharaevskii Yu.P., Serdobintsev A.A., Mitin D.M., Nikitov S.A. // Phys. Rev. Lett. 2018. Vol. 120. P. 257203.

[5] Damon R.W., Eshbach J.R. // J. Phys. Chem. Solids. 1961. Vol. 19. P. 308

[6] Gurevich A.G., Melkov G.A. Magnetization Oscillations and Waves. London, N. Y:: CRC-Press, 1996. 464 p.

[7] Stancil D.D., Prabhakar A. Spin Waves: Theory and Applications. N. Y.: Springer, 2009. 348 p.

[8] Sodha M.S., Srivastava N.C. Microwave Propagation in Ferrimagnetics. N. Y.: Springer, 1981. 403 p.

[9] Вашковский А.В., Стальмахов В.С., Шараевский Ю.П. Магнитостатические волны в электронике сверхвысоких частот. Саратов: СГУ, 1993. 310 с.

[10] Chikazumi S. Physics of Ferromagnetism. 2nd ed. Oxford: Oxford University Press, 1997. 682 p.

[11] Clausen P., Vogt K., Schultheiss H., Schäfer S., Obry B. // Appl. Phys. Lett. 2011. Vol. 99. P. 162505.

[12] Sadovnikov A.V., Davies C.S., Kruglyak V.V., Romanenko D.V., Grishin S.V., Beginin E.N., Sharaevskii Y.P., Nikitov S.A. // Phys. Rev. B. 2017. Vol. 96. P. 60401.

[13] Brächer T., Pirro P., Westermann J., Sebastian T., Lägel B., Van de Wiele B., Vansteenkiste A., Hillebrands B. // Appl. Phys. Lett. 2013. Vol. 102. P. 132411.

[14] Demokritov S. Spin Wave Confinement: Propagating Waves. 2nd Edition. Jenny Stanford Publishing, 2017. 436 p.

[15] Никитов С.А., Калябин Д.В., Лисенков И.В., Славин А.Н., Барабаненков Ю.Н., Осокин С.А., Садовников А.В., Бегинин Е.Н., Морозова М.А., Шараевский Ю.П., Филимонов Ю.А., Хивинщев Ю.В., Высочкий С.Л., Сахаров В.К., Павлов Е.С. // УФН. 2015. Т. 185. С. 1099-1128.

[16] Гуляев Ю.В., Никитов С.А. // ДАН. Сер. физ. 2001. Т. 380. C. 469.

[17] Kashyap R., Bragg F. Gratings. San Diego: Academic Press, 1999. P. 457. 
[18] Садовников А.В., Грачев А.А., Одинцов С.А., Мартышкин А.A., Губанов В.A., Шешукова С.E., Никитов С.А. // Письма в ЖЭТФ. 2018. Т. 108. № 5. C. 332 [Sadovnikov A.V., Grachev A.A., Odintsov S.A., Martyshkin A.A., Gubanov V.A., Sheshukova S.E., Nikitov S.A. // JETP Lett. 2018. 108:5. P. 312-317].

[19] Sadovnikov A.V., Beginin E.N., Morozova M.A., Sharaevskii Yu.P., Grishin S.V., Sheshukova S.E., Nikitov S.A. // App. Phys. Lett. 2016. Vol. 109. 042407.

[20] Морозова М.А, Матвеев О.В., Шараевский Ю.П. // ФТТ. 2016. Т. 58. Вып. 10. С. 1899-1906.

[21] Dvornik M., Au. Y., Kruglyak V.V. Micromagnetic Simulations in Magnonics. Ch. 8. Topics in Applied Physics. Vol. 125. S.O. Demokritov and A.N. Slavin Editors Magnonics From Fundamentals to Applications.

[22] Remouche M., Georges F., Meyrueis P. // Opt. Photon. J. 2012. Vol. 2. P. 1-7.

[23] Gaididei Y., Kravchuk V.P., Mertens F.G., Pylypovskyi O.V., Saxena A., Sheka D.D., Volkov O.M. // Low Temp. Phys. 2018. Vol. 44. P. 634.

[24] Tkachenko V.S., Kuchko A.N., Kruglyak V.V. // Low Temp. Phys. 2013. Vol. 39. P. 163.

[25] Bance S., Schrefl T., Hrkac G., Goncharov A., Allwood D.A., Dean J. // J. Appl. Phys. 2008. Vol. 103. P. 07E735.

[26] Ландау Л.Д. Электродинамика сплошных сред. М.: Наука, 1982. C. 621.

[27] Ландау Л.Д., Лифиии Е.M. // Phys. Zs. Sowjet. 1935. Vol. 8. P. $153-169$.

[28] Vansteenkiste A., Leliaert J., Dvornik M., Helsen M., GarciaSanchez F., Van Waeyenberge B. // AIP Adv. 2014. Vol. 4. P. 107133.

[29] Demidov V.E., Urazhdin S., Zholud A., Sadovnikov A.V., Demokritov S.O. // Appl. Phys. Lett. 2015. Vol. 106. P. 022403.

[30] Demokritov S.O., Hillebrand B., Slasvin A.N. // Phys. Rep. 2001. Vol. 348. P. 441.

[31] Sadovnikov A.V., Davies C.S., Grishin S.V., Kruglyak V., Romanenko D.V., Sharaevskii Yu.P., Nikitov S.A. // Appl. Phys. Lett. 2015. Vol. 106. P. 192406.

[32] Sadovnikov A.V., Odintsov S.A., Beginin E.N., Sheshukova S.E., Nikitov S.A. // Phys. Rev. B. 2017. Vol. 96. P. 144428.

[33] O’Keefe T.W., Patterson R.W. // J. Appl. Phys. 1978. Vol. 49. P. 4886. 